\title{
Effects of Acute Alcohol Consumption on the Human Brain: Diffusional Kurtosis Imaging and Arterial Spin-Labeling Study
}

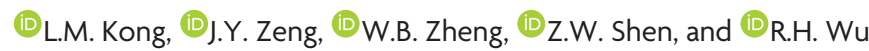

\begin{abstract}
BACKGROUND AND PURPOSE: Brain function and microstructure are affected by alcohol consumption. Until recently, the effect of alcohol on neural mechanisms has not been fully elucidated. Our aim was to explore the acute effects of alcohol on healthy human brains by diffusional kurtosis imaging and 3D arterial spin-labeling and elucidate structural and functional changes in the brain on acute alcohol intake.
\end{abstract}

MATERIALS AND METHODS: Conventional MR imaging, diffusional kurtosis imaging, and 3D arterial spin-labeling were performed on 24 healthy volunteers before and 0.5 and 1 hour after drinking alcohol. Participants were divided into 2 groups according to the response to alcohol: blushing $(n=12)$ and unblushing $(n=12)$ groups. Twenty brain regions were analyzed.

RESULTS: Diffusional kurtosis imaging revealed an increase in mean kurtosis and fractional anisotropy at 0.5 hour post-alcohol intake in most brain regions, whereas mean diffusion was decreased in several brain regions at 1 hour after drinking. 3D arterial spin-labeling showed increased cerebral blood flow in most brain regions, particularly in the frontal regions. However, perfusion in the anterior commissure decreased. Regional changes in the brain correlated with various behavioral performances with respect to blush response and sex. In general, blushing individuals and men are more sensitive to alcohol with acute effects.

CONCLUSIONS: Physiologic and microstructural alterations in the brain on alcohol consumption were examined. Brain areas with blood flow alteration detected by 3D arterial spin-labeling were highly consistent with susceptible areas detected by diffusional kurtosis imaging. The current study provides new insight into the effects of alcohol on the brain and behavioral performance in different blush response and sex populations.

ABBREVIATIONS: $A$ LDH = aldehyde dehydrogenase; $\mathrm{ASL}=$ arterial spin-labeling; $\mathrm{BAES}=$ Biphasic Alcohol Effects Scale; DKI = diffusional kurtosis imaging; $\mathrm{FA}=$ fractional anisotropy; $\mathrm{MD}=$ mean diffusion; $\mathrm{MK}=$ mean kurtosis; pre = controls

S tudies of acute alcohol challenge have revealed the neural and behavioral impairments that accompany intoxication. Alcohol affects multiple neurotransmitter systems in the brain and brain functions. ${ }^{1,2}$ Acute alcohol consumption has marked effects on brain metabolism and produces functional and morphologic

Received September 16, 2018; accepted after revision January 22, 2019.

From the Department of Radiology (L.M.K., J.Y.Z., W.B.Z., Z.W.S., R.H.W.), the Second Affiliated Hospital, Medical College of Shantou University, Shantou, China; and China Provincial Key Laboratory of Medical Molecular Imaging (R.H.W.), Guangdong, Shantou, China.

This study was supported by the National Natural Science Foundation of China (grant No. 81471730, 31870981, 81571627); Medical Scientific Research Foundation of Guangdong Province, China (grant No. B2014284); Shantou University “Innovation Powerhouse" Project, Training Program of Guangdong Province, China (No. 92338040239), and Shantou University Student, Innovation and Entrepreneurship Training Program of Guangdong Province, China (No. 201610560111).

Please address correspondence to Renhua Wu, MD, PhD, Department of Radiology, the Second Affiliated Hospital, Medical College of Shantou University, Shantou 515041 China; e-mail: rhwu@stu.edu.cn

-- Indicates open access to non-subscribers at www.ajnr.org

= Indicates article with supplemental on-line tables.

http://dx.doi.org/10.3174/ajnr.A5992 changes. Until recently, the effect of alcohol on neural mechanisms has not been fully elucidated. Alcohol abuse and dependence are a huge societal and economic burden that results in lost productivity, increased health care costs, and traffic accidents and contributes to domestic violence. The economic, social, and health consequences related to the consumption of drugs and alcohol are a global concern with $0.8 \%$ of the overall causes in global disability-adjusted life years attributable to illicit drugs and alcohol. $^{3}$

There are several types of treatment for alcohol use disorders; however, the mechanistic physiologic substrates of treatment remain to be elucidated. ${ }^{4}$ Alcohol dehydrogenase catalyzes the conversion of ethanol to acetaldehyde, which is further converted to acetic acid by aldehyde dehydrogenase (ALDH). Individuals carrying the $A L D H 22^{*}$ allele of the $A L D H 2$ gene produce an inactive ALDH2 enzyme resulting in excessive acetaldehyde production. ${ }^{6,7}$ Subsequent to alcohol consumption, the accumulation of acetaldehyde relaxes the capillaries resulting in facial blushing. Individuals carrying the $A L D H 2^{\star} 2$ allele may also have rapid heartbeat, headache, dizziness, sweating, nausea, or vomiting. Al- 
cohol-related facial blushing is an indicator of an inherited variant enzyme, aldehyde dehydrogenase, that impairs alcohol metabolism and increases the drinker's lifetime risk of certain aerodigestive cancers. Thus, it is suggested that people who blush should stop drinking permanently. ${ }^{8}$

Brain function and microstructure are affected by alcohol consumption. Our previous diffusion tensor imaging study showed alterations in brain tissue diffusion after acute alcohol intake, which underscores the susceptibility of neural systems to acute alcohol ingestion. ${ }^{9}$ Diffusional kurtosis imaging (DKI) quantifies non-Gaussian diffusion and has been suggested to be advantageous over DTI and better characterizes both normal and pathologic brain tissue, particularly for the assessment of gray matter. ${ }^{10}$ One of the primary indices of DKI, mean kurtosis (MK), measures the degree of diffusion restriction and indicates microstructural complexity. ${ }^{11}$ DKI has proved useful in diagnosing numerous cerebral pathologies such as congenital sensorineural hearing loss, ${ }^{12}$ gliomas, ${ }^{13}$ and acute cerebral infarction. ${ }^{14}$ Arterial spin-labeling (ASL), on the other hand, uses magnetically labeled arterial blood as an endogenous tracer to measure regional cerebral blood flow. While brain perfusion abnormalities might exist before morphologic changes, we hypothesized that the effects of acute alcohol on the physiology and structure of the brain are better determined by DKI and ASL.

The purpose of this study was to evaluate the feasibility of DKI and ASL to evaluate effect of acute alcohol intake on brain and to investigate both sex and blush effects.

\section{MATERIALS AND METHODS \\ Subjects}

Twenty-four healthy right-handed volunteers (12 men, 12 women; 23-27 years of age) with no history of alcohol or drug abuse were recruited for this study. They were light-moderate drinkers who reported drinking occasionally (mean, $3 \pm 0.8$ times per year). All procedures were performed in accordance with the ethical standards of the Second Affiliated Shantou Medical University Hospital and institutional review board. Written informed consent was obtained from all subjects before participation. Participants were familiarized with the setup and the scanner before the first experimental session. To ensure that the participants were not under the influence of alcohol, we requested that they abstain from alcohol for 24 hours and refrain from food for 6 hours before each experimental session.

\section{Study Protocols}

A dose of $0.65 \mathrm{~g}$ of alcohol per kilogram of body weight was given orally in the form of spirit (53 $3^{\circ}$ Maotai spirit, 2016; Maotai, Renhuai, Guizhou, China). Alcohol consumption was calculated on the basis of weight with the following equation: Alcohol Consumption (Milliliters) = Amount of Alcohol Intake (Grams) / Alcohol Concentration (Percentage) $\times 0.8$ (Ethanol Attenuation). Subjects were allowed to drink along with eating some snacks, such as peanuts. The alcohol was consumed in 6-10 minutes. Because no electronic devices could be used inside the scanner room, we measured breath alcohol concentrations, an index to estimate blood alcohol concentration, using a hand-held breathalyzer before the subjects entered the scanner (about 29 minutes, 59 minutes) and after taking them out of the scanner (about 40 minutes, 70 minutes). Heart rate and blood pressure were also measured. Participants were divided into 2 groups according to the response to alcohol: blushing ( $n=12,6$ men, 6 women) and unblushing ( $n=12,6$ men, 6 women). Subjective responses to alcohol were measured using the Biphasic Alcohol Effects Scale (BAES) ${ }^{15}$ at approximately 30 minutes before drinking and again at 25 and 55 minutes after the initiation of alcohol consumption.

\section{MR Imaging Protocols}

T2-weighted imaging, DKI, and 3D-arterial spin-labeling were acquired using a 3T MR imaging system (Signa; GE Healthcare, Milwaukee, Wisconsin) with an 8-channel head coil (HD 8Ch HiRes BRAIN ARRAY, GE Healthcare). Echo-planar imaging was used for DKI acquisition with the following parameters: TR/ $\mathrm{TE}=4500 / 98.6 \mathrm{~ms}$; slice thickness $=4 \mathrm{~mm}$ with a 1-mm gap; $\mathrm{FOV}=240 \times 240 \mathrm{~mm}$; matrix $=256 \times 256$; average $=1$; and $\mathrm{b}$-values $=0,1000,2000 \mathrm{~s} / \mathrm{mm}^{2}$ in 15 diffusion-encoding directions; imaging time $=3$ minutes, 5 seconds. ASL was performed with the following parameters: TR/TE $=4580 / 9.8 \mathrm{~ms}$; 4-mm slice thickness with no gap; FOV $=240 \times 240 \mathrm{~mm}$; postlabeling delay time $=1.5$ seconds; average imaging time $=4$ minutes, 26 seconds. Foam pads were used to reduce head movement. Subjects were asked to lie still with their eyes closed during data acquisition.

\section{Data Analysis}

All data were transferred to the workstation (Advantage Workstation 4.6; GE Healthcare) and FuncTool software package (GE Healthcare) for data processing. DKI metrics, including fractional anisotropy (FA), mean diffusivity (MD), and MK, were derived by a research tool in the FuncTool environment developed by the GE Applied Science Lab (see http://www.nitrc.org/projects/dke/).

Twenty brain regions (superior frontal gyrus, middle frontal gyrus, inferior frontal gyrus, precentral gyrus, postcentral gyrus, frontal white matter, anterior cingulate cortex, posterior cingulate cortex, cuneus cortex, occipital gyrus, superior temporal gyrus, hippocampal gyrus, parahippocampal gyrus, lenticular nucleus, dorsal thalamus, hypothalamus, amygdaloid body, anterior commissure, posterior commissure, and cerebellar hemisphere) were manually delineated by 2 experienced radiologists (5-6 years' experience) for ROI analysis (sample images in Fig 1), in which each ROI was approximately $12 \mathrm{~mm}^{2}$. Both hemispheres were analyzed, which resulted in 40 ROIs. The DKI and ASL values for the ROIs were acquired and averaged over 3 replicates by every radiologist and were averaged by 2 surveyors to correct for inter- and intraobserver error.

\section{Statistical Analysis}

All statistical analyses were performed with SPSS 20.0 for Windows (IBM, Armonk, New York). Data were analyzed using univariate repeated-measures analysis of variance for the elimination of individual differences. To compare the difference in MK, FA, and $\mathrm{MD}$, we used BAES ratings in the control (pre), 0.5 hour, and 1-hour time points and used a repeat 1-way analysis of variance test. If the overall test of the 2 means for the MK, FA, MD, and 


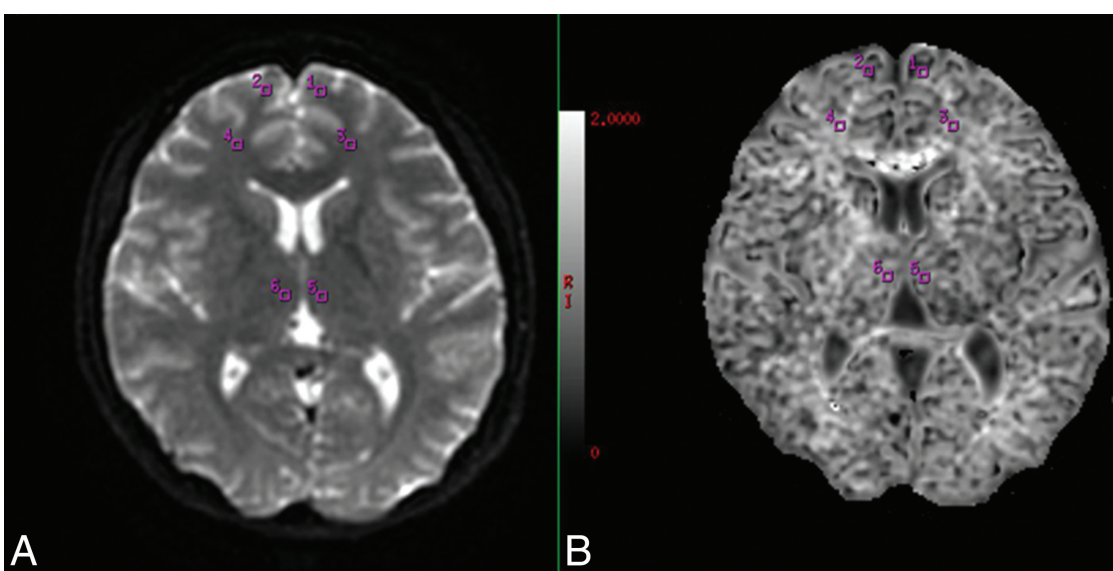

FIG 1. Example of the selection of ROIs from $3 / 20$ brain regions under study. $A$, The raw DKI map. $B$, The gray level of the MK map. Two radiologists manually drew the ROIs on the raw DKI map and then obtained the MK values automatically. For ASL, the ROls were also drawn on the raw ASL map manually and then the CBF values were obtained automatically.

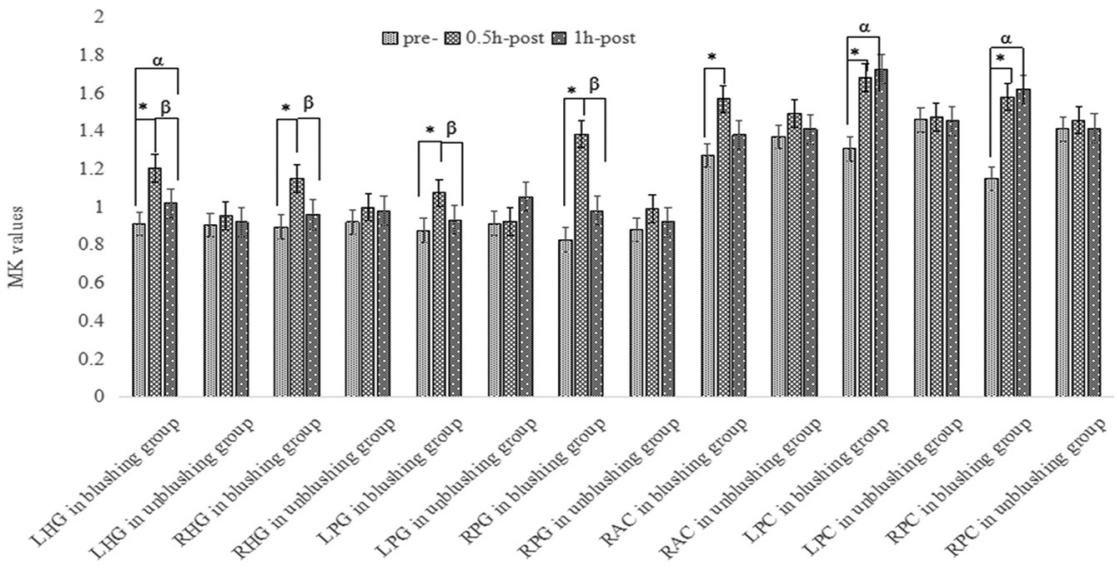

FIG 2. Blushing effect in MK at different time points. LHG indicates left hippocampal gyrus; RHG, right hippocampal gyrus; LPG, left parahippocampal gyrus; RPG, right parahippocampal gyrus; RAC, right anterior commissure; LPC, left posterior commissure; RPC, right posterior commissure; asterisk, a statistically significant difference between 0.5 hour and pretreatment $(P<.05) ; \alpha$, a statistically significant difference between 1 hour and pretreatment $(P<.05) ; \beta$, a statistically significant difference between 1 hour and 0.5 hour $(P<.05)$; pre, before; post, after.

increased speech were seen in women more than in men at 0.5 hour. However, these more severe symptoms in women had apparently improved at 1 hour. Online Table 1 shows the mean (SD) of baseline ratings for the BAES items at different time points.

Breath alcohol concentration and heart rate increased and peaked at 0.5 hour after alcohol consumption, then decreased to baseline. There were no significant changes in blood pressure. No correlation was found among changes in breath alcohol concentration, heart rate, or blood pressure index or blushing (On-line Table 2).

\section{MR Imaging}

Conventional T2WI showed no observable differences in signal intensities between pre- and post-alcohol consumption. MK increased and peaked at 0.5 hour in 38/40 ROIs and remained elevated in 31/40 ROIs at 1 hour compared with baseline. A delayed decrease in MD was observed, in which decreased MD was found in 3/40 ROIs at 0.5 hour, and 18/40 ROIs at 1 hour following alcohol consumption compared with baseline. An increase in FA was found in 23/40 ROIs at 0.5 hour. FA in 36/40 ROIs returned to baseline, whereas 4/40 ROIs remained elevated at 1 hour. ASL indicated increased CBF in 36/40 ROIs at 0.5 hour after alcohol consumption. This effect declined dramatically, with CBF returning to baseline at 1 hour. Most interesting, bilateral anterior commissure CBF decreased at 0.5 hour compared with baseline. CBF in the left pre-

BAES ratings values showed statistically significant differences, then multiple comparisons of the means of any 2 groups were performed using the Bonferroni post hoc test. A Wilcoxon rank sum test for continuous variables was used to determine the intraand interobserver variability in MK, FA, and MD. All measurements were expressed as means \pm SDs. A $P$ value $<.05$ was considered statistically significant.

\section{RESULTS}

\section{Clinical Manifestations}

Symptoms at 0.5 hour after alcohol consumption included headache $(n=16)$, nausea and vomiting $(n=16)$, dizziness $(n=15)$, walking unsteadily $(n=12)$, blushing $(n=12)$, increased speech $(n=11)$, excitement $(n=10)$, dysphoria $(n=9)$, depressed mood $(n=9)$, feeling tired $(n=6)$, and sleepiness $(n=5)$. Symptoms were generally improved at 1 hour. Some symptoms (dizziness, increased speech, excitement, and dysphoria) were more long-lasting and stronger in blushing than nonblushing individuals. Nausea and vomiting were more severe and greater excitement and central and right inferior frontal gyri increased, whereas CBF in the left anterior commissure was decreased at 1 hour compared with baseline. MK, MD, FA, and CBF values for 20 different brain regions are summarized in On-line Table 3. Moreover, MK and CBF alterations were found in both white and gray matter.

\section{Blush Effect}

At 0.5 hour, MK in the bilateral hippocampal gyrus, parahippocampal gyrus and posterior commissure, and right anterior commissure was increased for blushing individuals but not for nonblushing individuals (Fig 2). At 0.5 hour, MD was increased in the left anterior commissure for blushing individuals but not for nonblushing individuals. At 1 hour, MK in the right superior frontal gyrus, left parahippocampal gyrus, and posterior commissure was increased in the blushing group. At 1 hour, MD in the right middle frontal gyrus decreased in the blushing group, whereas decreased MD in the superior temporal gyrus was found in the nonblushing group. 


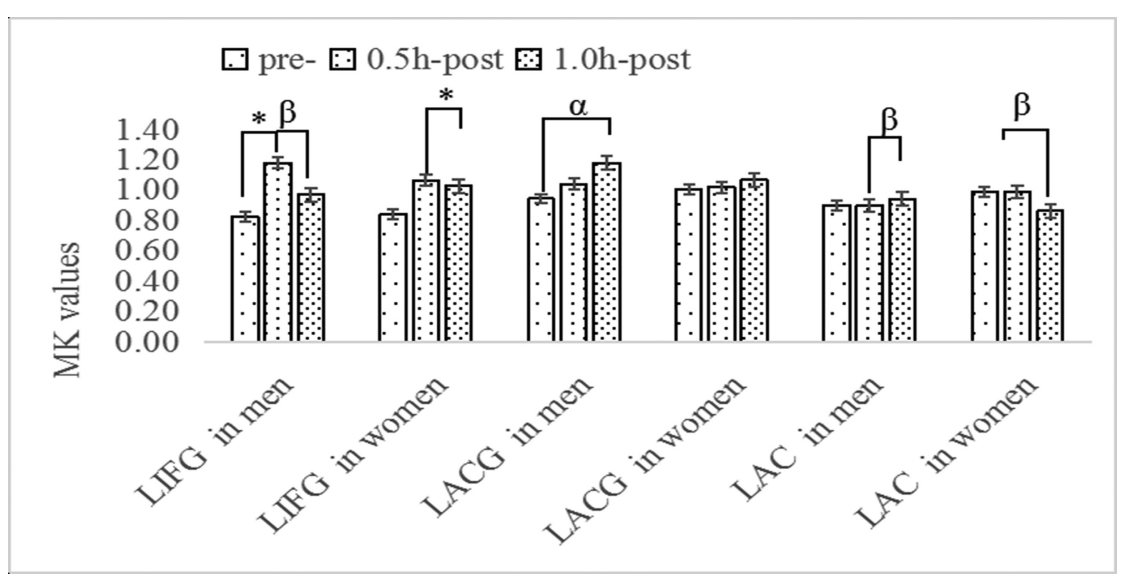

FIG 3. Sex effects in MK at different time points. LIFG indicates left inferior frontal gyrus; LACG, left anterior central gyrus; LAC, left anterior cingulate; asterisk, a statistically significant difference between 0.5 hour and pretreatment $(P<.05) ; \alpha$, a statistically significant difference between 1 hour and pretreatment $(\mathrm{P}<.05) ; \beta$, a statistically significant difference between 1 hour and 0.5 hour $(P<.05)$; pre, before; post, after.

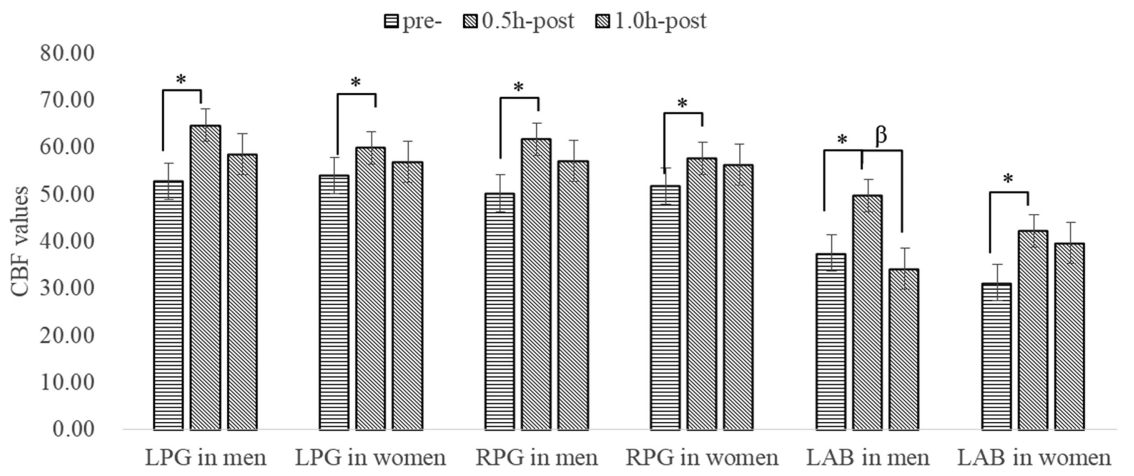

FIG 4. Sex effect in CBF at different time points. LPG indicates left parahippocampal gyrus; RPG, right parahippocampal gyrus; $L A B$, left amygdaloid body; asterisk, a statistically significant difference between 0.5 hour and pretreatment $(P<.05) ; \beta$, a statistically significant difference between 1 hour and 0.5 hour $(P<.05)$; pre, before; post, after.

At 1 hour compared with 0.5 hour, MK was significantly decreased in the left frontal white matter, hippocampal gyrus, right dorsal thalamus, and parahippocampal gyrus in the blushing group, whereas MK was increased in the nonblushing group. We observed increased MK in the right parahippocampal gyrus in the blushing group but not in the nonblushing group. CBF appeared higher in the superior temporal gyrus in blushing individuals at 0.5 hour.

\section{Sex Effect}

MK in the left inferior frontal gyrus showed a larger increase in men than in women at 0.5 hour, and a larger increase in the left anterior central gyrus was found in men at 1 hour. At 1 hour compared with 0.5 hour, MK in left inferior frontal gyrus decreased more in men. MK in the left anterior cingulate in women decreased, whereas it increased in men (Fig 3). At 1 hour, MD in the left dorsal thalamus decreased more in men than in women. Figure 4 shows a larger increase in CBF in men than in women in the bilateral parahippocampal gyrus and left amygdaloid body at 0.5 hour. Moreover, CBF returned to baseline values in the left amygdaloid body in men yet remained elevated in women at 1 hour.

\section{DISCUSSION}

The current study investigated the physiologic and microstructural alterations in the brain on alcohol consumption. MD was decreased in several brain regions at 1 hour after drinking. Some studies suggest that reduction in MD could be related to cytotoxic edema, whereas an elevation in MD indicates vasogenic edema. ${ }^{16}$ Cytotoxic edema may occur after alcohol consumption, which is in line with prior studies in both humans and animals. ${ }^{9,17,18}$ Yet, another study reported no changes in diffusion parameters measured using diffusion MR imaging in acute alcohol intake. ${ }^{19}$ On the other hand, DKI revealed an increase in MK and FA at 0.5 hour postalcohol intake in most brain regions. This increase in MK could be related to cell swelling during cytotoxic edema, during which the space between myelin fiber bundles is reduced and the volume fraction of restricted water diffusion is increased. ${ }^{20}$ Thus, the extracellular tortuosity and microstructural complexity of the organization is likely increased and reflected by an increase in MK and FA. The alteration of microstructural complexity is related to brain edema, suggestive of direct toxicity. Increased diffusional kurtosis in the brain could be associated with decreased membrane permeability $^{21}$ and cell swelling during cytotoxic edema in specific brain regions. The results suggested that edema may be more severe in the blushing groups, translating into potential neurologic injury and impaired function of these brain regions. MK is altered at 0.5 hour after alcohol consumption, whereas MD changes occurred at 1 hour. Moreover, alterations in MK were detected in numerous brain regions compared with MD. These may indicate that MK has a higher sensitivity to detect microstructural changes in both white matter and gray matter after acute alcohol intake.

The increased CBF detected in most brain regions, particularly in the frontal regions, was consistent with that in previous studies using ASL. ${ }^{22,23}$ This finding could be because alcohol increases the generation of reactive oxygen species (such as nitric oxide and lipid peroxidation products) ${ }^{24}$ and augments endothelium-dependent vasodilation. ${ }^{25}$ In particular, the effect of alcohol on perfusion might be related to its metabolic effect and might be dominated by the direct vasodilatory effects of alcohol. ${ }^{23}$ The coupling between neuronal activity and CBF could also be disrupted by biphasic vasoactive properties of alcohol. ${ }^{26}$ Although acetaldehyde, a metabolite of alcohol, acts as a vasodilator, high concentrations of alcohol constrict blood vessels depending on calcium ions. Global changes in CBF are an important marker of cerebral 
autoregulation, and increased CBF in the sensory and motor areas may be related to the disinhibitory effects of alcohol on related functions. ${ }^{27}$ The return of CBF to baseline at 1 hour may reflect compensation. However, perfusion in the anterior commissure decreased. One explanation for such a decrease might be the impairment in autoregulation of brain blood flow. ${ }^{28}$ The anterior commissure is an attenuated white matter structure with little vasculature. The blood flow of this deep white matter structure may decrease relative to the increase of cortical perfusion. Acute alcohol intake exerts differential effects in various brain regions. ${ }^{29,30}$

Our findings of both DKI and ASL imply that the thalamus, limbic system, cerebellum, and especially the frontal lobes are more subject to alcohol-related brain damage than other cerebral regions. We hypothesize that alcohol-induced microstructural changes in these sensitive brain regions, which are associated with the function of these regions, likely result in both physiologic and psychological consequences on behavior. The limbic system primarily includes the nucleus accumbens, amygdala, and hypothalamus and predominantly controls appropriate responses to stimuli with social, emotional, or motivational salience, ${ }^{31}$ it is involved in the regulation of sensorial and visceral activity and closely related to psychological activities such as learning, memory, the generation of emotional responses, and behavior. Alcohol-induced parameter changes in the thalamus were associated with changes in mood. These alcohol-induced changes in mood have also been predicted by changes in thalamic connectivity, whereas changes in motor performance have been associated with the cerebellothalamic network. ${ }^{32}$ The prefrontal cortex is thought to be one of the most complex anatomic and functional structures of the human brain. Acute ethanol administration in humans has been shown to cause deficits in executive activities and language performance associated with the prefrontal cortex. The deleterious effects of acute ethanol administration on prefrontal cortex-dependent behaviors may result from the breakdown of functional specificity in different brain regions. $^{33}$

The blush effect of the brain was investigated by DKI and ASL. To our knowledge, blush effects have not been previously examined by these techniques. Blushing and nonblushing individuals showed differential changes in brain regions, which could be related to emotional, neuropsychological, and language-expression changes in the 2 groups. Alcohol-use disorders may induce alterations in gene expression, including $A L D H$, in brain regions such as the prefrontal cortex, ${ }^{34}$ and the superior frontal gyrus is involved in a variety of functions, such as execution ${ }^{35}$ and language functions. ${ }^{36}$ The parahippocampal gyrus plays a key role in the information flow between cortical association areas and the hippocampal formation, ${ }^{37}$ and this region may reflect particular sensitivity to the subjective emotional state during emotional regulation. ${ }^{38}$ Increased MK at 1 hour in the right superior frontal gyrus of blushing individuals might mean that these individuals were easily affected in language expression and execution, whereas increased MK in the left parahippocampal gyrus in the nonblushing group may be associated with the lag of emotional and neuropsychological function. In combination with our data on CBF, these findings further suggest that alcohol exerts regional effects on the brain and that the regional distribution of CBF might reflect alcohol effects on functional brain activity. ${ }^{39}$
Cognitive-behavioral abilities are affected differently by ethanol. Not all regions of the brain had significant differences in DKI parameters and CBF values at 0.5 - and 1.0-hour time points in the blushing-versus-nonblushing group. Regional $\mathrm{MK}$ and $\mathrm{CBF}$ changes could be associated with clinical symptoms. MR imaging studies of sex differences in brain function of alcoholics have yielded contradictory results, with some studies showing women to be more susceptible than men to brain impairment and vice versa. ${ }^{32,40}$ In general, we found more microstructural changes in the left inferior frontal gyrus, amygdaloid body, precentral gyrus, anterior cingulate, and bilateral parahippocampal gyrus in men. One plausible explanation is that a few brain regions of men are more susceptible to alcohol, which manifests in changes in language expression, executive function, memory, and emotion of men after alcohol consumption. Moreover, the primary somatic motor center and limbic system of men are more likely to be affected across time with a longer duration, which may impact limb movement.

Apart from microstructural changes, sex effects in brain perfusion changes remain controversial. Increased frontal perfusion in men was reported by Rickenbacher et $\mathrm{al},{ }^{41}$ whereas Marxen et $\mathrm{al}^{23}$ detected a significantly higher brain perfusion in women. The discrepancies between these studies and ours might be related to the difference in alcohol dosage and the time course of imaging. Additional imaging studies with larger samples, together with age, blushing, sex, population, breath alcohol concentration, alcohol history, and different time points after drinking are needed to sort out the effects of alcohol intoxication. A multivariate analysis might be beneficial to account for demographic and physiologic covariates.

This study has several limitations. More subjects are needed to further investigate the potential effects of blushing, sex, and the time course of diffusion and perfusion responses. In particular, the current study only investigated the effect of acute alcohol (within 1 hour) consumption with a few time points. More time points and longer time spans are required to better investigate the changes of brain function with time. Last, subjects recruited in this study were young adults. More age groups, including adolescents, middle-aged adults, and the elderly, will be included in future studies.

\section{CONCLUSIONS}

The extent of alteration in DKI and ASL parameters in various brain areas may be useful in quantifying the extent of damage to brain tissue. People who consume alcohol on a regular basis with imperceptible changes in cognitive abilities, DKI, ASL, and cognitive test findings will be measured regularly (for example, yearly) in a future study. The correlation of DKI and ASL parameters to cognitive function will be examined to determine the most relevant indicators of decrements in cognitive function. Because blushing individuals are more sensitive to alcohol with acute effects, people who blush should stop drinking on the basis of current findings. The current study demonstrated that brain areas with blood flow alterations detected by 3D-ASL were highly consistent with susceptible areas detected by DKI after acute alcohol intake. Regional changes in the brain vary with respect to 
blush response and sex. Blushing individuals and men are more sensitive to alcohol with acute effects.

\section{ACKNOWLEDGMENTS}

The authors thank Yuelin Guo, MD, PhD, in the Department of Radiology, the Second Affiliated Hospital, Medical College of Shantou University, Guangdong, China, for technologic assistance.

Disclosures: Wenbin B. Zheng-RELATED: Grant: National Natural Science Foundation of China.* Renhua H. Wu—RELATED: Grant: National Natural Science Foundation of China. * Money paid to the institution.

\section{REFERENCES}

1. Mukherjee S, Das SK, Vaidyanathan K, et al. Consequences of alcohol consumption on neurotransmitters: an overview. Curr Neurovasc Res 2008;5:266-72 CrossRef Medline

2. White AM. What happened? Alcohol, memory blackouts, and the brain. Alcohol Res Health 2003;27:186-96 Medline

3. Hoang VP, Shanahan M, Shukla N, et al. A systematic review of modelling approaches in economic evaluations of health interventions for drug and alcohol problems. BMC Health Serv Res 2016;16: 127 CrossRef Medline

4. Nielsen AS, Nielsen B. Implementation of a clinical pathway may improve alcohol treatment outcome. Addict Sci Clin Pract 2015;10:7 CrossRef Medline

5. de la Monte SM, Kril JJ. Human alcohol-related neuropathology. Acta Neuropathol 2014;127:71-90 CrossRef Medline

6. Helminen A, Väkeväinen S, Salaspuro M. ALDH2 genotype has no effect on salivary acetaldehyde without the presence of ethanol in the systemic circulation. PLoS One 2013;8:e74418 CrossRef Medline

7. Pavlova SI, Jin L, Gasparovich SR, et al. Multiple alcohol dehydrogenases but no functional acetaldehyde dehydrogenase causing excessive acetaldehyde production from ethanol by oral streptococci. Microbiology 2013;159(Pt 7):1437-46 CrossRef Medline

8. Ding LY, Yuen LW, Newman IM, et al. University students' willingness to assist fellow students who experience alcohol-related facial flushing to reduce their drinking. Int J Environ Res Public Health 2018;15 CrossRef Medline

9. Kong LM, Zheng WB, Lian GP, et al. Acute effects of alcohol on the human brain: diffusion tensor imaging study. AJNR Am J Neuroradiol 2012;33:928-34 CrossRef Medline

10. Jensen JH, Helpern JA. MRI quantification of non-Gaussian water diffusion by kurtosis analysis. NMR Biomed 2010;23:698-710 CrossRef Medline

11. Van Cauter S, Veraart J, Sijbers J, et al. Gliomas: diffusion kurtosis MR imaging in grading. Radiology 2012;263:492-501 CrossRef Medline

12. Zheng WB, Wu CX, Huang LX, et al. Diffusion kurtosis imaging of microstructural alterations in the brains of paediatric patients with congenital sensorineural hearing loss. Sci Rep 2017;7:1543 CrossRef Medline

13. Bai Y, Lin Y, Tian J, et al. Grading of gliomas by using monoexponential, biexponential, and stretched exponential diffusion-weighted MR imaging and diffusion kurtosis MR imaging. Radiology 2016;278:496504 CrossRef Medline

14. Guo YL, Zhang ZP, Zhang GS, et al. Evaluation of mean diffusion and kurtosis MRI mismatch in subacute ischemic stroke: comparison with NIHSS score. Brain Research 2016;1644:231-39 CrossRef Medline

15. Rueger SY, McNamara PJ, King AC. Expanding the utility of the Biphasic Alcohol Effects Scale (BAES) and initial psychometric support for the Brief-BAES (B-BAES). Alcohol Clin Exp Res 2009;33: 916-24 CrossRef Medline

16. Cernak I, Vink R, Zapple DN, et al. The pathologies of moderate diffuse traumatic brain injury as identified using a new experimental model of injury in rats. Neurobiol Dis 2004;17:29-43 CrossRef Medline

17. Liu HM, Zheng WB, Yan G, et al. Acute ethanol-induced changes in edema and metabolite concentrations in rat brain. Biomed Res Int 2014;2014:351903 CrossRef Medline

18. Chen XR, Zeng JY, Shen ZW, et al. Diffusion kurtosis imaging detects microstructural changes in the brain after acute alcohol intoxication in rats. Biomed Res Int 2017;2017:4757025 CrossRef Medline

19. Duninga T, Kugelc H, Menkea R, et al. Diffusion-weighted magnetic resonance imaging at 3.0 Tesla in alcohol intoxication. Psychiatry Res 2008;163:52-60 CrossRef Medline

20. Sotak $\mathrm{CH}$. The role of diffusion tensor imaging in the evaluation of ischemic brain injury: a review. NMR Biomed 2002;15:561-69 CrossRef Medline

21. Jensen JH, Helpern JA, Ramani A, et al. Diffusional kurtosis imaging: the quantification of non-gaussian water diffusion by means of magnetic resonance imaging. Magn Reson Med. 2005;53: 1432-40 CrossRef Medline

22. Tolentino NJ, Wierenga CE, Hall S, et al. Alcohol effects on cerebral blood flow in subjects with low and high responses to alcohol. $\mathrm{Al}$ cohol Clin Exp Res 2011;35:1034-40 CrossRef Medline

23. Marxen M, Gan G, Schwarz D, et al. Acute effects of alcohol on brain perfusion monitored with arterial spin-labeling magnetic resonance imaging in young adults. J Cereb Blood Flow Metab 2014;34: 472-79 CrossRef Medline

24. Zahr NM, Kaufman KL, Harper CG. Clinical and pathological features of alcohol-related brain damage. Nat Rev Neurol 2011;7: 284-94 CrossRef Medline

25. Puddey IB, Zilkens RR, Croft KD, et al. Alcohol and endothelial function: a brief review. Clin Exp Pharmacol Physiol 2001;28: 1020-24 CrossRef Medline

26. Kawano Y. Physio-pathological effects of alcohol on the cardiovascular system: its role in hypertension and cardiovascular disease. Hypertens Res 2010;33:181-91 CrossRef Medline

27. Rose AK, Duka T. The influence of alcohol on basic motoric and cognitive disinhibition. Alcohol Alcohol 2007;42:544-51 CrossRef Medline

28. Jochum T, Reinhard M, Boettger MK, et al. Impaired cerebral autoregulation during acute alcohol withdrawal. Drug Alcohol Depend 2010;110:240 - 46 CrossRef Medline

29 Grundersen H. van Wageningen H, Grüner R. Alcohol-induced changes in cerebral blood flow and cerebral blood volume in social drinkers. Alcohol Alcohol 2013;48:160-65 CrossRef Medline

30. Bjorka JM, Gilman JM. The effects of acute alcohol administration on the human brain: insights from neuroimaging. Neuropharmacology 2014;84:101-10 CrossRef Medline

31. Sokolowski K, Corbin JG. Wired for behaviors: from development to function of innate limbic system circuitry. Front Mol Neurosci 2012;5:55 CrossRef Medline

32. Shokri-Kojori E, Tomasi D, Wiers CE, et al. Alcohol affects brain functional connectivity and its coupling with behavior: greater effects in male heavy drinkers. Mol Psychiatry 2017;22:1185-95 CrossRef Medline

33. Abernathy K, Chandler LJ, Woodward JJ. Alcohol and the prefrontal cortex. Int Rev Neurobiol 2010;91:289-320 CrossRef Medline

34. Zhang HP, Wang F, Xu HQ, et al. Differentially co-expressed genes in postmortem prefrontal cortex of individuals with alcohol use disorders: influence on alcohol metabolism-related pathways. Hum Genet 2014;133: 1383-94 CrossRef Medline

35. Li W1, Qin W, Liu H, et al. Subregions of the human superior frontal gyrus and their connections. Neuroimage 2013;78:46-58 CrossRef Medline

36. Fujii M, Maesawa S, Motomura K, et al. Intraoperative subcortical mapping of a language-associated deep frontal tract connecting the superior frontal gyrus to Broca's area in the dominant hemisphere of patients with glioma. J Neurosurg 2015;122:1390-96 CrossRef Medline

37. Echávarri C, Aalten $\mathrm{P}$, Uylings $\mathrm{HB}$, et al. Atrophy in the parahippocampal gyrus as an early biomarker of Alzheimer's disease. Brain Struct Funct 2011;215:265-71 CrossRef Medline 
38. Frank DW, Dewitt M, Hudgens-Haney M, et al. Emotion regulation: quantitative meta-analysis of functional activation and deactivation. Neurosci Biobehav Rev 2014;45:202-11 CrossRef Medline

39. Khalili-Mahani N, van Osch MJ, Baerends E, et al. Pseudocontinuous arterial spin-labeling reveals dissociable effects of morphine and alcohol on regional cerebral blood flow. J Cereb Blood Flow Metab 2011;31:1321-33 CrossRef Medline
40. Ide JS, Zhornitsky S, Hu S, et al. Sex differences in the interacting roles of impulsivity and positive alcohol expectancy in problem drinking: a structural brain imaging study. Neuroimage Clin 2017; 14:750-59 CrossRef Medline

41. Rickenbacher E, Greve DN, Azma S, et al. Effects of alcohol intoxication and gender on cerebral perfusion: an arterial spin-labeling study. Alcohol 2011;45:725-37 CrossRef Medline 